\title{
A New Approach Layered Architecture based Clustering for Prolong Life of Wireless Sensor Network (WSN)
}

\author{
Babu Ram, Narottam Chand, Prateek Gupta, Siddhartha Chauhan \\ Department of computer science \& engineering \\ National Institute Of Technology-Hamirpur \\ Hamirpur (H.P.) India
}

\begin{abstract}
Sensor nodes are the tiny particles which have to rely on limited power of energy. Sensor nodes with limited battery power are deployed to gather information in wireless environment throughout the field. Due to the small amount of energy in a node of sensor web, energy balancing among nodes is quite important. Secondly, due to lack of energy in a particular node we are also concerned to the power saving of a sensor node. We are also concerned here to prolong the life of sensor node so that it can sense the field for longer period of time. All these features are the prime necessity to introduce a new energy efficient routing algorithm in this paper.
\end{abstract}

\section{Keywords}

Wireless Sensor Network, layered architecture, data collecting range, transmission range

\section{I- INTRODUCTION}

Low cost inexpensive sensors which are capable of significant computation are become available [1,2]. Sensor networks are comprise of many different types of sensor nodes which are capable of Environment monitoring, such as temperature, humidity, lighting, noise levels, pressure, the presence or absence of particular objects and the object properties such as speed, direction and size. A network of wireless sensor nodes can be deployed in the field to get useful information [3]. Sensor nodes are highly constrained by the amount of battery power, limiting the lifetime of the network [12, 13]. As concerned to the wireless communication it uses major amount of battery power so sensor nodes should spend a little amount of energy for receiving and transmitting data $[4,5$, and 6]. It is necessary for the protocols to maximize the nodes lifetime $[7,14]$ and reduce the bandwidth consumption by the collaboration among the nodes and tolerate node failures [8].

\section{II- RELATED WORK}

LEACH is based on the fusion (or aggregation) technique which combines the original data into the smaller size of data and carries only meaningful information to all the sensor nodes [9]. In case of LEACH, cluster heads are not selected statically, as by this they will lose their energy and die quickly. Instead of this LEACH uses the rotational policy so that all the sensor nodes one by one will act as the cluster head by this the energy of every node will equally diminish and avoid the battery depletion of an individual sensor node. Here cluster heads are selected with some probability and broadcast their status among all other sensors node in the network. Any sensor node can become cluster head without any negotiation with other sensor nodes. A sensor decides to become a cluster head based on the desired percentage $\mathrm{P}$ of cluster heads, the current round, and the set of sensors that have not become cluster heads in the past $1 / \mathrm{P}$ rounds. If the number of cluster heads is $<\mathrm{T}(\mathrm{n})$, a sensor node $\mathrm{n}$ will become a cluster head for the current round, where $\mathrm{T}(\mathrm{n})$ is a threshold as,

$$
T(n)= \begin{cases}\frac{p}{1-P\left(r \bmod \frac{1}{p}\right)} & \text { if } n \in G, \\ 0 & \text { otherwise. }\end{cases}
$$

LEACH enables data fusion by aggregating the data in each cluster to reduce the total amount of data before sending to the sink. In other words, cluster head aggregates the data from all the sensor nodes and then transmits to the sink. PEGASIS another protocol which is the improvement over LEACH was proposed in $[10,11]$ that allow only one cluster head to transmit to the sink in each round. However, a sensor node has to transmit the data to its local neighbor in the data fusion phase instead of sending data directly to its cluster head as in the case of LEACH protocol. In PEGASIS, sensor nodes are arranged in the form of chain, which is formed either by the sensor nodes itself using greedy algorithm or by the sink. In the construction phase it is assumed that all the sensor nodes have the global knowledge about the network. When a sensor node dies due to the low battery, the chain is constructed by bypassing the dead sensor node. The chain has two end sensor nodes and in each data fusion phase only one leader will transmit the data to the sink. Any other intermediate sensor node will fuse the data that will be received from the neighbor with its own data and forward the fused data to its neighbor located nearer to the sink instead of itself so that the data get forwarded to the sink.

\section{III-PROBLEM STATEMENT}

When the network is first time deployed, the energy of all the nodes is same. As the network starts functioning, few nodes lose their energy faster and die earlier as compared to other nodes. The main problem is that how to manage the energy consumption among the nodes so that less energy could be consumed among nodes and we can increase the lifetime of the network. We cannot replace the battery of the sensor nodes as it is not easy task that's why we have to make the network so that the energy of all the nodes will remain balance by which we can prolong the life of the network. 


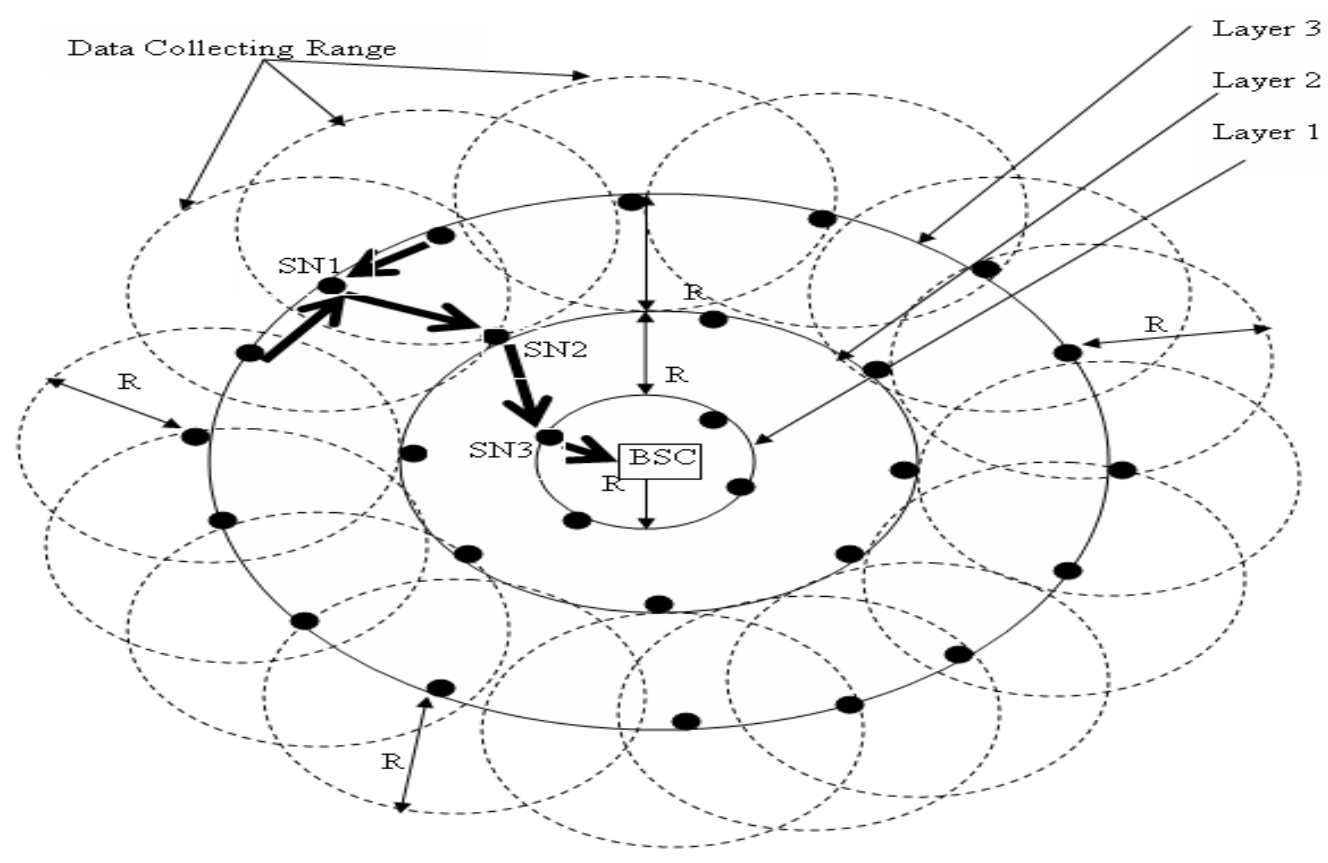

Fig 1: Layered Architecture Based Clustering

\section{IV-PROPOSED SOLUTION}

Wireless Sensor Network has a sink node also called as Base Station Controller (BSC). BSC has no condition of battery power and computational limitation. In our proposed solution we are using layered architectural approach. Sensor nodes are distributed among these layers according to the distance from the BSC and each layer is consisting of many sensor nodes. Each layer is at $\mathrm{R}$ distance from the previous layer. Layer 1 is at $\mathrm{R}$ distance from the BSC, Layer 2 is at R distance from the first layer, Layer 3 is at $\mathrm{R}$ distance from the second layer. The main purpose of layered approach is to communicate and send the data of higher level to the lower level sensor nodes using minimum transmission energy. All sensor nodes of higher level will communicate to the lower level sensor nodes which are at the minimum distance $\mathrm{R}$. As the transmission energy is directly proportional to the distance $\mathrm{R}$. $\mathrm{R}$ is the minimum distance between layers so it will take the minimum energy to transmit the data between different levels of the layers. The second concept we use in this paper is Data Collecting Range showed in the figure 1 with the dotted circle. These Data collecting Range will be defined at each layer. The radius of the Data collecting Range is R. All the sensor nodes in the data collecting range fuse their data at the sensor node which will at the maximum energy and forward the fused data to the next lower level as shown in the figure as dark black arrow. In the figure shown DCR1 is a data collecting range with radius $\mathrm{R}$ which consists of many sensor nodes and SN1 is the maximum energy node is DCR1 at layer 3. All the sensor nodes will send their collected data to the SN1 sensor node and SN1 will fuse the data and forward to the sensor node at maximum energy at the layer 2 . In our figure 1 SN2 is the maximum energy node. SN2 sensor node will forward the data to its inner level layer (layer 1) to SN3. Similarly, SN3 will forward the data to the Base Station
Controller (BSC). Distance R between each layer and the Radius $\mathrm{R}$ of Data Collecting Range depends on:
a. Battery Power of Wireless Sensor Node
b. Transmission Range of Wireless Sensor node.
c. Density of WSN per $\mathrm{m}^{2}$

\section{V-EXPERIMENTAL RESULT}

We have simulated the result of our proposed model, LEACH and PEGASIS in 100-nodes network using Omnetpp-4.1 version. Figure 1 shows 100 nodes network which is randomly deployed in the field. We have located BSC at the center of $50 \mathrm{~m} \times 50 \mathrm{~m}$ field. We simulated our result to determine the number of rounds of communication when $1 \%$, $25 \%, 50 \%, 75 \%$ and $100 \%$ of the nodes die using LEACH, PEGASIS and our proposed model with each node having same initial energy level.

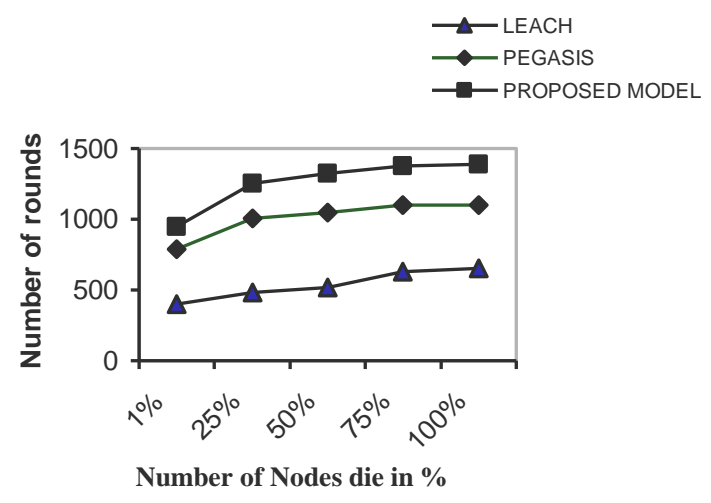

Fig 2: Experimental result for network with initial energy $0.25 \mathrm{~J} /$ node 


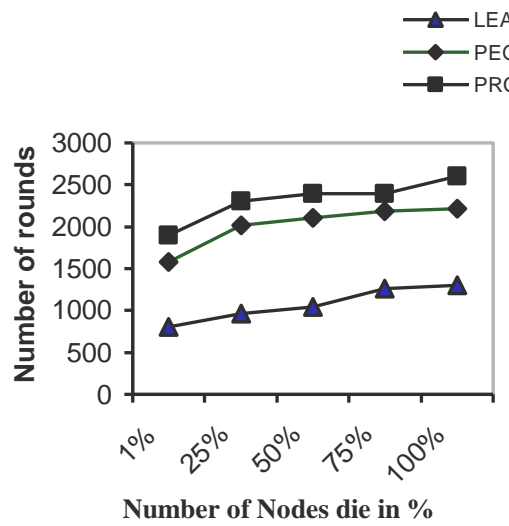

Fig 3: Experimental result for network with initial energy 0 $.5 \mathrm{~J} /$ node

$$
\begin{aligned}
& \longrightarrow \text { LEACH } \\
& \longrightarrow \text { PEGASIS } \\
& \longrightarrow \text { PROPOSED M ODEL }
\end{aligned}
$$

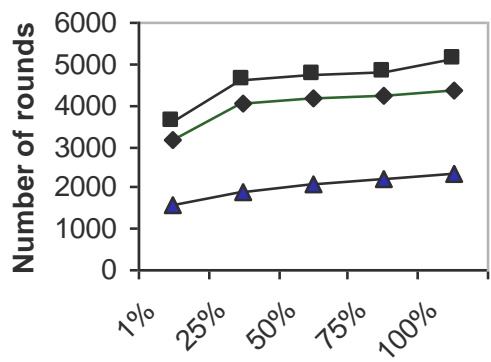

Number of Nodes die in \%

Fig 4: Experimental result for network with initial energy 1.0 $\mathrm{J} /$ node

Figure 2 shows the number of rounds until $1 \%, 25 \%, 50 \%$, $75 \%$ and $100 \%$ nodes die for a $50 \mathrm{~m} \times 50 \mathrm{~m}$ network with $0.25 \mathrm{~J}$ initial energy per node, $0.5 \mathrm{~J}$ per node in Figure 3 and $1.0 \mathrm{~J}$ per node in Figure 4. Our proposed model performs approximately $1.35 \mathrm{x}$ better than PEGASIS and approximately $4 \mathrm{x}$ better than LEACH

\section{VI- CONCLUSION}

In this paper, we have discussed the new proposed model which is based on dynamic data gathering approach in the formation of cluster head of sensor nodes to minimize the overhead and distance from BSC so that less dissipation of energy in sensor nodes at the time of data transmission. From our simulation results it is show that our proposed model is $135 \%$ better than PEGASIS and $400 \%$ better than LEACH when $1 \%, 25 \%, 50 \%, 75 \%$ and $100 \%$ of nodes die for the described network.

\section{VII-REFERENCES}

[1] D. Estrin, R Govindan, J. Heidemann, and Satish Kumar. Next Century Challenges: Scalable Coordination in Sensor Networks. In Proceedings of Mobicom ' 99, 1999.
[2] J. Kulik, W. Rabmer, and H. Balakrishnan. Adaptive Protocols for Infamation Dissemination in Wireless Sensor Networks. In Proceedings of Mobicom' 99, 1999.

[3] Tbe WINS Project. httu://www.ianet.ucla.edu/Wn\T.

[4] W. Mangione-Smith and P.S.Ghang. A Low Power Medium Access Control Protocol for Portable MultiMedia Systems. In Proceedings 3rd lntl. Workshop on Mobile Multimedia Communications. F'rinceton, NJ, Sept. 25-27, 1996.

[5] K. M. Sivalingam, M. B. Srivastava and P. Agrawal. Low Power Link and Access Protocols for Wireless Multimedia Networks. In Proceedings IEEE VehicularTechnology Conference VTC'97, May 1997.

[6] M. Stemm, P. Gauthier, D. Harada and R Katz.Reducing Power Consumption of Network Interfaces in Hand-Held Devices. In Proceedings 3rd Intl.Workshop on Mobile Multimedia Communications,Sept. 25-27, 1996 , Princeton, NJ.

[7] S. Sin\& M. Woo and C.S. Raghavendra. Power-Aware Routing in Mobile Ad Hoc Networks. In Proceedings ACMIEEE Mobicom 1998.

[8] M. Zorzi and R. R. Rao. Energy Management in Wireless Communications. In Proceedings $6^{\text {th }}$ WLIVLAB Workshop on Third Generation Wireless Information Nenvorkr, March 1997.

[9] Revision of Part 15 of the Commission' $s$ Rules Regarding Ultra - Wideband Transmission Systems. First note and Order, Federal Communications Commission, ET - Docket 98 - 153, Adopted Feb. 14, 2002, released Apr. 22, 2002.

[10] W. Heinzelman, A. Chandrakasan, and H. Balakrishnan, "Energy-efficient communication protocols for wireless microsensor networks,"Proceedings of the $33^{\text {rd }}$ InternationalConference on System Sciences, January 2000.

[11] W. Heinzelman, A. Chandrakasan, and H. Balakrishnan, "An application-specific protocol architecture for wireless microsensor networks, "IEEE Transactions on Wireless Communication, vol. 1, No. 4, pp. 660-670, October 2002.

[12] J. Yick, B. Mukherjee, and D. Ghosal. Wireless sensor network survey. Computer Networks, 52(12), 2008.

[13] A.A. Abbasi and M. Younis, A survey on clustering algorithms for wireless sensor networks, Elsevier Computer Communications 30 (14-15) (2007), pp. 28262841.

[14] Wei, D., Kaplan, S. and Chan H .A. Energy Efficient Clustering Algorithms for Wireless Sensor Networks. In Proceedings of IEEE Communications Society (ICC 2008), pp.236-240. 